\title{
Explicit LPV-MPC with Bounded Rate of Parameter Variation
}

\author{
Thomas Besselmann* Johan Löfberg ${ }^{* *}$ Manfred Morari ${ }^{* * *}$ \\ * Automatic Control Laboratory, ETH Zurich, Switzerland, (www: \\ http://control.ee.ethz.ch, e-mail: \\ besselmann@control.ee.ethz.ch) \\ ** Department of Electrical Engineering, Linköping University, \\ SE-581 83 Linköping, Sweden, (www: \\ http://www. control.isy.liu.se, e-mail: johanl@isy.liu.se) \\ *** Automatic Control Laboratory, ETH Zurich, Switzerland, (www: \\ http://control.ee.ethz.ch, e-mail: morari@control.ee.ethz.ch)
}

\begin{abstract}
Recently it was shown how one can reformulate the MPC problem for LPV systems to a series of mpLPs by a closed-loop minimax MPC algorithm based on dynamic programming. A relaxation technique is employed to reformulate constraints which are polynomial in the scheduling parameters to parameter-independent constraints. The algorithm allows the computation of explicit control laws for LPV systems and enables the controller to exploit information about the scheduling parameter. This improves the control performance compared to a standard robust approach where no uncertainty knowledge is used, while keeping the benefits of fast online computations. In this paper the explicit LPV-MPC scheme is extended to incorporate limits on the rate of parameter variation in the controller design. Taking these limits into account can further improve control performance since impossible trajectories of the scheduling parameter do not have to be considered during control law computations.
\end{abstract}

Keywords: Robust Model Predictive Control; Parametric Uncertainties; Computational Methods.

\section{INTRODUCTION}

The study of Linear Parameter-Varying (LPV) systems is motivated by their use in gain-scheduling control techniques for nonlinear systems, Shamma and Athans [1990, 1991], Apkarian et al. [1995]. Classical gain-scheduling approaches work with an interpolation of the controller gains among a family of linear time-invariant (LTI) controllers, which are based on linearized models of the system. While those techniques work surprisingly well in practice, it is hard to give precise stability/performance statements taking changes in the system dynamics into account. LPV systems account for changes in the system dynamics by parameter-varying system matrices. The parameters lie in a bounded set, such that an LPV system describes a family of linear systems. The LPV framework constitutes a useful theoretical foundation and allows statements on stability and performance which take variations of the scheduling parameter directly into account.

Linear parameter-varying systems were also considered in the Model Predictive Control (MPC) community, Lu and Arkun [2000], Chisci et al. [2003]. The introduction of multi-parametric programming into the field of model predictive control around the millennium allows the computation of explicit solutions for the optimal control problem of constrained linear and piecewise affine systems, Bank et al. [1982], Bemporad and Morari [1999], Pistikopoulos et al. [2000], Bemporad et al. [2002], Borrelli [2003]. Instead of solving an optimization problem at each sampling instance, the optimal input is obtained from a look-up table, which significantly reduces the online computational effort. In Bemporad et al. [2003], the computation of explicit control laws was extended to linear discrete-time systems with parametric uncertainty, i.e., when the parameter is bounded, but unknown. The computation of explicit control laws for LPV systems - when the scheduling parameter is known - was presented recently, Besselmann et al. [2008a,b].

The proposed procedure assumes the scheduling parameter to vary arbitrarily within a defined set. In practice however, there are often limitations on the rate of parameter variation. Two of many examples for LPV systems with limited parameter variations are the amount of fuel in an airplane or the engine speed engine in engine control systems. Taking these limitations not into account can result in overly conservative control. This fact has been recognized in the literature and is incorporated in other control methods for LPV systems, see e.g. Chisci et al. [2003]. In the present paper we are proposing an extension to the explicit LPV-MPC scheme, which enables the incorporation of limitations on the rate of parameter variation.

The paper is structured as follows: In Section 2, the considered problem is stated. The explicit LPV-MPC scheme is restated in Section 3, followed by the extensions for limited rate of parameter variations in Section 4 . A numerical example illustrates the application of the algorithm in Section 5. 


\subsection{Notation}

The set of non-negative real numbers is denoted by $\mathbb{R}_{+}$. The positive orthant in the $n$-dimensional Euclidean space is denoted by $\mathbb{R}_{+}^{n}$. A polyhedron is a set described by the intersection of finitely many half-spaces. A polytope is a closed and bounded polyhedron. The Minkowski sum of two sets is denoted by $\bigoplus$. An upper index in brackets denotes the element of a vector or the row in a matrix.

\section{PROBLEM STATEMENT}

We consider linear discrete-time LPV systems with a parameter-varying state transition and parameter-varying input matrix

$$
x_{k+1}=A\left(\theta_{k}\right) x_{k}+B\left(\theta_{k}\right) u_{k} .
$$

The variables $x_{k} \in \mathbb{R}^{n_{x}}, u_{k} \in \mathbb{R}^{n_{u}}$ and $\theta_{k} \in \mathbb{R}_{+}^{n_{\theta}}$ denote the state, control input and time-varying scheduling parameter, respectively. Furthermore, the system is constrained, $x_{k} \in \mathbb{X}$ and $u_{k} \in \mathbb{U}$. The constraint sets $\mathbb{X}$ and $\mathbb{U}$ are assumed to be polytopes including the origin in its interior

$$
\begin{aligned}
& \mathbb{X}=\left\{x: E_{x} x \leq f_{x}\right\}, \\
& \mathbb{U}=\left\{u: E_{u} u \leq f_{u}\right\} .
\end{aligned}
$$

Remark 1. We restrict ourselves to separate constraints on the state and inputs in (2) solely for ease of notation. It is straight-forward to modify the presented algorithm in this paper to the case of mixed constraints, i.e. $E_{x} x+$ $E_{u} u \leq f_{x u}$.

The scheduling parameter vector $\theta_{k}=\left[\theta_{k}^{[1]}, \ldots, \theta_{k}^{\left[n_{\theta}\right]}\right]^{T}$ is measured online. Future values are however not known precisely. Information available is that the future scheduling parameters are the barycentric coordinates of a standard $n_{\theta}$-simplex $\Theta$ in the parameter space,

$$
\Theta:=\left\{\theta_{k} \in \mathbb{R}_{+}^{n_{\theta}}: \sum_{j=1}^{n_{\theta}} \theta_{k}^{[j]}=1\right\},
$$

and that the rate of variation $\gamma$ is limited

$$
\gamma=\theta_{k+1}-\theta_{k} \in \Gamma \subset \mathbb{R}^{n_{\theta}}
$$

The set $\Gamma$ denotes all possible variations of the scheduling parameter within one time step.

The parameter-varying matrices $A\left(\theta_{k}\right)$ and $B\left(\theta_{k}\right)$ are known to lie in polytopes,

$$
A\left(\theta_{k}\right)=\sum_{j=1}^{n_{\theta}} A_{j} \theta_{k}^{[j]}, \quad B\left(\theta_{k}\right)=\sum_{j=1}^{n_{\theta}} B_{j} \theta_{k}^{[j]},
$$

where $A_{j}$ and $B_{j}$ denote the $j$-th vertices of the polytopes. This polytopic description is a common assumption in the LPV framework, see e.g. Apkarian et al. [1995]. For the control problem to make sense, it is assumed that the system (1) is controllable (and observable) for all $\theta_{k} \in \Theta$, see Silverman and Meadows [1967], Balas et al. [2003]. For this class of systems we want to compute an explicit statefeedback control law

$$
u_{k}=\mu_{k}\left(x_{k}, \theta_{k}\right) \text {, }
$$

which makes use of knowledge of the current scheduling parameter $\theta_{k}$. In order to compute this control law (6) within a Model Predictive Control scheme, a cost function is to be minimized. The control objective is to stabilize the LPV system (1) to the origin. According to standard $\mathrm{MPC}$, our cost function is defined as

$$
J=\left\|P x_{k+N}\right\|_{p}+\sum_{i=0}^{N-1}\left\|Q x_{k+i}\right\|_{p}+\left\|R u_{k+i}\right\|_{p},
$$

where $p$ denotes a piecewise linear norm, either the 1norm or the $\infty$-norm. Piecewise linear norms ${ }^{1}$ enable a parametric solution to the stated problem using dynamic programming. For the minimization of the cost function (7) we have to consider the current as well as the unknown future scheduling parameter values, as the state trajectories are parameter-dependent.

\section{EXPLICIT MPC FOR LPV SYSTEMS}

For the sake of completeness, this section restates the computation of explicit MPC controllers for LPV systems, Besselmann et al. [2008b]. Before starting the actual computation of the control law (6), the polynomial dependency of the control law $\mu$ on the scheduling parameter has to be decided. This dependency can chosen to be affine

$$
\mu_{k}\left(x_{k}, \theta_{k}\right)=\sum_{j=1}^{n_{\theta}} \theta_{k}^{[j]} \mu_{k}^{j}\left(x_{k}\right),
$$

but note that in principle any polynomial in the parameter $\theta_{k}$ is possible with our proposed method. In the case of the affine parametrization (8), the function $\mu_{k}^{j}\left(x_{k}\right)$ corresponds to the control law in the $j$-th vertex of the parameter simplex (3). In order to simplify notation, we introduce the basis $U_{k}:=\left\{\mu_{k}^{1}, \mu_{k}^{2}, \ldots, \mu_{k}^{n_{\mu}}\right\}$.

In a closed-loop MPC approach, one would assume that the future control law $\mu_{k+i}$ is calculated optimally over the horizon $N-i$ not until $x_{k+i}$ and $\theta_{k+i}$ are available. But as the future values of the scheduling parameters are unknown, all possible cases must be considered in order to accommodate for the worst-case scenario. This way constraint satisfaction is assured and the actual cost function will be less or equal to the computed one, no matter how the scheduling parameters evolve. The optimization problem to solve in closed-loop minimax MPC is thus

$$
\mu_{k}\left(x_{k}, \theta_{k}\right)=\arg \min _{U_{k}} \min _{U_{k+1}} \max _{\theta_{k+1}} \cdots \min _{U_{k+N-1}} \max _{\theta_{k+N-1}} J
$$

Here we propose a dynamic programming (DP) procedure to solve (9) by iterating backwards in time. For more details on dynamic programming, see Bertsekas [1995]. We start at the prediction horizon $N$ with the initial cost function

$$
J_{N}^{*}\left(x_{k+N}\right)=\left\|P x_{k+N}\right\|_{p} .
$$

Then at each iteration we use

$$
x_{k+i+1}=A\left(\theta_{k+i}\right) x_{k+i}+B\left(\theta_{k+i}\right) \mu_{k+i}\left(x_{k+i}, \theta_{k+i}\right)
$$

to substitute $x_{k+i+1}$ in $J_{i+1}^{*}\left(x_{k+i+1}\right)$. As $\theta_{k+i}$ is unknown at time instance $k$, we consider the worst case, which leads to

$$
\begin{aligned}
J_{i}^{*}\left(x_{k+i}\right) & =\min _{U_{k+i}} \max _{\theta_{k+i}}\left\|Q x_{k+i}\right\|_{p}+\left\|R u_{k+i}\right\|_{p} \\
& +J_{i+1}^{*}\left(x_{k+i+1}\right) .
\end{aligned}
$$

1 Quadratic cost functions are not possible since our procedure relies on epigraph reformulations, which would render the original problem a multiparametric quadratically constrained quadratic program, for which no efficient solution techniques are available. 
In order to determine the worst-case parameters of (12), we first apply an epigraph reformulation to the optimization problem in order to transfer the parameter dependence to the constraints. This leads to the following semi-infinite optimization problem

$$
J_{i}^{*}\left(x_{k+i}\right)=\min _{U_{k+i}} t
$$

$$
\begin{aligned}
& \text { s.t. } \forall \theta_{k+i} \in \Theta: \\
& \left\|Q x_{k+i}\right\|_{p}+\left\|R u_{k+i}\left(\theta_{k+1}\right)\right\|_{p}+ \\
& J_{i+1}^{*}\left(A\left(\theta_{k+i}\right) x_{k+i}+B\left(\theta_{k+i}\right) \mu_{k+i}\left(x_{k+i}, \theta_{k+i}\right)\right) \leq t, \\
& x_{k+i} \in \mathbb{X}, \quad u_{k+i} \in \mathbb{U} .
\end{aligned}
$$

Remark 2. Depending on the structure of (11), the assurance of constraint satisfaction differs. If the control law (6) is chosen to be parameter-independent, the constraints in (13) are convex in the scheduling parameters and the maximum is attained at one of the vertices of the parameter simplex $\Theta$. However, the resulting control law does not take the current scheduling parameters into account, Bemporad et al. [2003]. For a constant input matrix $B$, it is reasonable to assume a polytopic input parametrization. The resulting constraints depend affinely on the scheduling parameter, which again allows for vertex enumeration. This case was tackled in detail in Besselmann et al. [2008a].

In the more general case of a non-constant input matrix $B\left(\theta_{k}\right)$ and a polynomially parameterized input $u_{k}\left(\theta_{k}\right)$, the constraints are polynomial in the scheduling parameters and a vertex enumeration is not sufficient to ensure constraint satisfaction over the whole simplex. However, the constraint satisfaction of the semi-infinite optimization problem (13) can be ensured, conservatively, over the whole parameter simplex with Pólya's theorem:

Theorem 1. Pólya's theorem. If a homogeneous polynomial $p(\theta)$ is positive on the simplex $\Theta$, all the coefficients of $p_{N_{p}}(\theta)=p(\theta) \cdot\left(\sum_{j=1}^{n_{\theta}} \theta^{[j]}\right)^{N_{p}}$ are positive for a sufficiently large Pólya degree $N_{p}$.

Proof 1. See Pólya [1928], Powers and Reznick [2001].

We will make use of the more obvious reverse of Pólya's theorem $^{2}$, i.e., positive coefficients of the extended polynomial mean positivity over the whole simplex.

The following design procedure describes the relaxation of the parameter-dependent constraints of (13) into constraints which are piecewise affine in the state and inputs and independent of the scheduling parameter:

(1) Reformulate constraints which are polynomial in the scheduling parameter into a positivity constraint of a polynomial $p(\theta)$.

(2) Homogenize the polynomial $p(\theta)$ by multiplying single monomials with $\sum_{j=1}^{n_{\theta}} \theta^{[j]}(=1)$ until all monomials have the same degree.

(3) Set the Pólya degree $N_{p}$, and compute the coefficients $c_{N_{p}}$ of the extended polynomial $p_{N_{p}}(\theta)=p(\theta)$. $\left(\sum_{j=1}^{n_{\theta}} \theta^{[j]}\right)^{N_{p}}$. In this step some conservatism may be introduced depending on the selection of $N_{p}$. By increasing the polynomial degree $N_{p}$, the relaxations become tighter until the exact problem is considered.

2 The presented usage of Pólya's theorem is implemented in YALMIP as one of the so called filters in the robust optimization framework, Löfberg [2008].
If all coefficients $c_{N_{p}}$ are non-negative, so is the polynomial $p(\theta)$. Hence the semi-infinite optimization problem (13) can be transformed into the following multi-parametric linear program (mp-LP):

$$
\begin{gathered}
J_{i}^{*}\left(x_{k+i}\right)=\min _{U_{k+i}} t \\
\text { s.t. } \quad c_{N_{p}}\left(x_{k+i}, U_{k+i}, t\right) \geq 0, \quad x_{k+i} \in \mathbb{X}
\end{gathered}
$$

Note that the coefficients of the extended polynomial lie in the cone which is spanned by the coefficients of the polynomial constraints in (13), and the piecewise affine dependence of the coefficients on the state is preserved. By using piecewise linear norms instead of quadratic norms, the cost functions $J_{i}^{*}$ are piecewise linear functions of the state $x_{k+i}$, such that in every iteration the optimization problem (13) can be formulated as the multi-parametric linear program (14) and solved parametrically with respect to $x_{k+i}$. Contrary to the closed-loop minimax MPC approach for uncertain systems, the future inputs are functions of the future scheduling parameters.

The final step of the DP procedure differs from the preceding steps, since knowledge of the current scheduling parameter values can be exploited to improve control performance. We make use of the uncontrolled successor state (USS),

$$
z_{k}=\left(\sum_{j=1}^{n_{\theta}} A_{j} \theta_{k}^{[j]}\right) x_{k},
$$

which was introduced in Besselmann et al. [2008a]. By parameterizing the parametric problem not in the measured state $x_{k}$, but in the USS, the parameter dependence of $A\left(\theta_{k}\right)$ can directly be taken into account. In lack of an equivalent scheme for the input, we minimize the worstcase gain-scheduled cost, i.e. solve the semi-infinite optimization problem

$$
J^{*}\left(z_{k}\right)=\min _{U_{k}} t+s
$$

s.t. $\forall \theta_{k} \in \Theta$ :

$$
\begin{gathered}
\left\|R \mu_{k}\left(z_{k}, \theta_{k+1}\right)\right\|_{p}+J_{1}^{*}\left(z_{k}+B\left(\theta_{k}\right) \mu_{k}\left(z_{k}, \theta_{k}\right)\right) \leq t \\
u_{k} \in \mathbb{U}, \\
\epsilon \sum_{j=1}^{n_{\theta}}\left\|Q\left(z_{k}+B\left(\theta_{k}^{[j]}\right) \mu_{k}\left(z_{k}, \theta_{k}^{[j]}\right)\right)\right\|_{p} \leq s,
\end{gathered}
$$

employing an additional epigraph variable $s$. For many problems, the vertex solutions are not unique, which can lead to irregular control laws when solving the parametric problem, and therefore a small regularization weight, $0<$ $\epsilon \ll 1$, which penalizes the vertex predictions, is added as an ad-hoc measure. While this virtually does not change the shape of the cost function, it turns out that the actual achieved performance is improved, because the non-uniqueness of the vertex solutions is mitigated. The polynomial dependence on the scheduling parameter can again be treated by employing Pólya's theorem to transform (16) into an mp-LP. The resulting control law $\mu_{k}\left(z_{k}, \theta_{k}\right)$ is piecewise affine in the USS, defined over a set of polytopes in the USS-space, and polynomial in the scheduling parameter, such that is can be written as

$$
\mu_{k}\left(z_{k}, \theta_{k}\right)=F_{r}\left(\theta_{k}\right) z_{k}+g_{r}\left(\theta_{k}\right) \quad \text { if } \quad z_{k} \in \mathbb{D}_{r}
$$

where 


$$
\mathbb{D}_{r}=\left\{z: E_{r} z \leq f_{r}\right\} \quad r=\left\{1, \ldots, n_{r}\right\} .
$$

When applying the computed control law online, in each step the state $x_{k}$ and the scheduling parameter $\theta_{k}$ are measured and used to compute the uncontrolled successor state $z_{k}$, which is then inserted in the parametric solution to obtain the control input $u_{k}$.

\section{BOUNDED RATE OF VARIATION}

In the following we will extend the results from the preceding section to incorporate limits on the rate of parameter variation in the controller computations.

The main drawback of explicit MPC is the inherent complexity of the resulting control laws. The driving factors for complexity (i.e. the number of regions) are the dimension of the state vector, the prediction horizon and the dimension of the scheduling parameter vector. When the complexity of the parametric solution is too high, evaluating the control law can become as time-consuming as solving the optimization problem online, hence explicit MPC techniques should only be applied to relatively small systems. Already for a linear prediction model it is hard to define 'relatively small systems' precisely since the complexity is highly depending on the application at hand. But to get a flavor of what relatively small means, one can observe that many applications of explicit MPC employ prediction models of state dimension five or lower, while only in exceptions the dimension is higher than ten, Hovland and Gravdahl [2008].

It is obvious that replacing a linear prediction model by an LPV model, i.e. including a scheduling parameter, will increase the complexity of the resulting control laws. Following the complexity argument above one has to assume reasonably that explicit LPV-MPC will primarily be applied to LPV models with a low dimensional scheduling parameter vector.

Assumption 1. The dimension $n_{\theta}$ of the parameter simplex $\Theta$ is low enough to apply Delaunay triangulations, which would be prohibitive in higher dimensions..

When the rate of parameter variation is limited, it follows that, starting from a certain initial scheduling parameter value, not all points in the parameter simplex can be attained in the succeeding time steps, but depending on time, some parts of the parameter simplex can be excluded. Hence different parts of the parameter simplex should be considered individually, which leads to the central point of this paper: The adaptation of the dynamic programming procedure in Section 3 for the computation of control laws for separate subsets of the parameter simplex $\Theta$. This adaptation firstly involves the determination of appropriate subsets of the parameter simplex $\Theta$ for each time step of the DP procedure.

(1) Partition the parameter simplex $\Theta$ into sub-simplices $\left\{\Theta_{1}, \ldots \Theta_{c}, \ldots, \Theta_{n_{s}}\right\}$.

It is required that these simplices are full-dimensional, collectively exhaustive,

$$
\bigcup_{c=1}^{n_{c}} \Theta_{c}=\Theta
$$

and mutually exclusive,

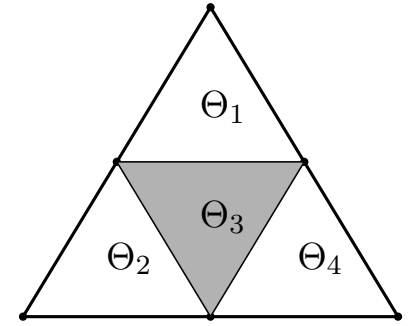

(a) Possible partition into four sub-simplices.

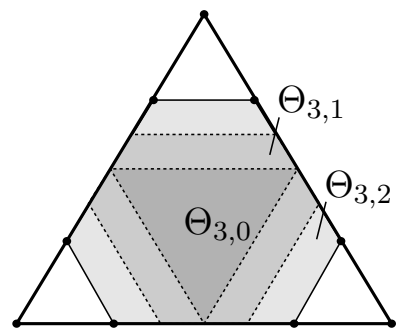

(b) Evolution of the simplex $\Theta_{3}$ over two time-steps.

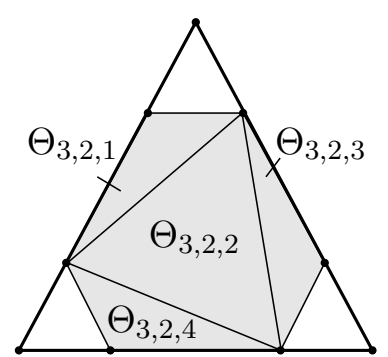

(c) Delaunay triangulation of polytope $\Theta_{3,2}$.

Fig. 1. Determination of the sub-simplices $\Theta_{c, i, s}$ of the 2dimensional parameter simplex $\Theta$.

$$
\Theta_{c_{1}} \bigcap \Theta_{c_{2}}=\emptyset \quad \forall c_{1} \neq c_{2} .
$$

A possible partition of a 2-dimensional parameter simplex into four simplices is depicted in Figure 1(a). Note that the partition is not required to be symmetric but can be adapted to the needs of the application at hand.

(2) Determine the evolution of each sub-simplex $\Theta_{c}$ over the prediction horizon, $\left\{\Theta_{c, 0}, \ldots, \Theta_{c, i}, \ldots, \Theta_{c, N}\right\}$.

Each simplex $\Theta_{c}$ represents one initial region $\Theta_{c, 0}$ for the scheduling parameter. Due to the limitation of the rate of parameter variation, the future parameters $\theta_{k+i}$, starting from the simplex $\Theta_{c, 0}$, have to lie within

$$
\Theta_{c, i}=\left(\Theta_{c, i-1} \bigoplus \Gamma\right) \bigcap \Theta .
$$

Figure 1(b) depicts the evolution of the simplex $\Theta_{3}$ over two prediction steps.

(3) Triangulate each polytope $\Theta_{c, i}$ to obtain the simplices $\left\{\Theta_{c, i, 1}, \ldots, \Theta_{c, i, s}, \ldots, \Theta_{c, i, n_{s, c}, i}\right\}$.

The application of Pólya's theorem as shown in Section 3 requires the scheduling parameter to live in the standard simplex. For this purpose the polytopes $\Theta_{c, i}$ at each prediction step are divided by a Delaunay triangulation, Delaunay [1934], resulting in a number $n_{s, c, i}$ of simplices. Each simplex $\Theta_{c, i, s}$ can then be transformed to the standard simplex by a coordinate transformation. The Delaunay triangulation of polytope $\Theta_{3,2}$ is shown in Figure 1(c).

Remark 3. Step 3) is not necessary when we are dealing with an LPV-A system with constant input matrix as in Besselmann et al. [2008a], since in that case the application of Pólya's theorem simplifies to a vertex enumeration.

With all subsets $\Theta_{c, i, s}$ of the parameter simplex $\Theta$ at hand, we can start the actual computation of the LPVMPC control law. For every sub-simplex $\Theta_{c}$ a separate parameter-affine control law is computed using a modified version of the DP procedure in Section 3. In the following 
we will focus on the required modifications for the normal DP steps starting from (13). These modifications have to be applied in an analogous fashion to the final DP step (16).

Taking a limited rate of variation into account means instead of solving the semi-infinite optimization problem (13) at the $(N-i)$ th step of the DP procedure, one would rather solve

$$
\begin{aligned}
& \text { s.t. } \forall \theta_{k+i} \in \Theta_{c, i}: \\
& \left\|Q x_{k+i}\right\|_{p}+\left\|R u_{k+i}\left(\theta_{k+1}\right)\right\|_{p}+ \\
& J_{i+1}^{*}\left(A\left(\theta_{k+i}\right) x_{k+i}+B\left(\theta_{k+i}\right) \mu_{k+i}\left(x_{k+i}, \theta_{k+i}\right)\right) \leq t, \\
& x_{k+i} \in \mathbb{X}, \quad u_{k+i} \in \mathbb{U} .
\end{aligned}
$$

In the case that $\Theta_{c, i}$ is not a simplex, each sub-simplex $\Theta_{c, i, s}$ from the Delaunay triangulation is treated separately,

$$
J_{i}^{*}\left(x_{k+i}\right)=\min _{U_{k+i}} t
$$

$$
\begin{aligned}
& \text { s.t. } \forall s: \forall \theta_{k+i} \in \Theta_{c, i, s}: \\
& \left\|Q x_{k+i}\right\|_{p}+\left\|R u_{k+i}\left(\theta_{k+1}\right)\right\|_{p}+ \\
& J_{i+1}^{*}\left(A\left(\theta_{k+i}\right) x_{k+i}+B\left(\theta_{k+i}\right) \mu_{k+i}\left(x_{k+i}, \theta_{k+i}\right)\right) \leq t, \\
& \quad x_{k+i} \in \mathbb{X}, \quad u_{k+i} \in \mathbb{U},
\end{aligned}
$$

by transforming it to the standard simplex and applying Pólya's theorem.

The online application of the computed control law now consists of the following steps: (i) measure or estimate the state $x_{k}$ and the scheduling parameter $\theta_{k}$, (ii) identify the sub-simplex $\Theta_{c}$ to which $\theta_{k}$ belongs, (iii) compute the USS $z_{k}$ and (iv) determine the control law related to $\Theta_{c}$ by evaluating the parametric solution.

Remark 4. Taking the rate of parameter variation into account results in an increase of complexity (approximately by the number of sub-simplices, $n_{c}$ ). Hence the determination of an appropriate number $n_{c}$ remains to be a trade-off between control performance and complexity. Note however that the additional complexity is affecting the online computation time far less than the storage space. Step (iv) takes approximately the same amount of time as before, and only step (ii) is additionally to the case with unlimited rate of variation.

Remark 5. The presented modification of the explicit LPV-MPC scheme can also be used to generalize the originally investigated case of unlimited rate of variation. It allows for applications where the scheduling parameter is not living in a standard simplex, but in a general polytope. Instead of increasing the dimension of the parameter simplex for each additional vertex of the polytope by one, one can partition the polytope into sub-simplices and apply the procedure above.

Remark 6. Performance benefits are not only due to considering the limitations on the rate of variation, but also to the richer structure of the resulting control law. Instead of being an affine (/polynomial) function of the scheduling parameter, the dependence of the control law is piecewise affine (/polynomial), and not necessarily continuous.

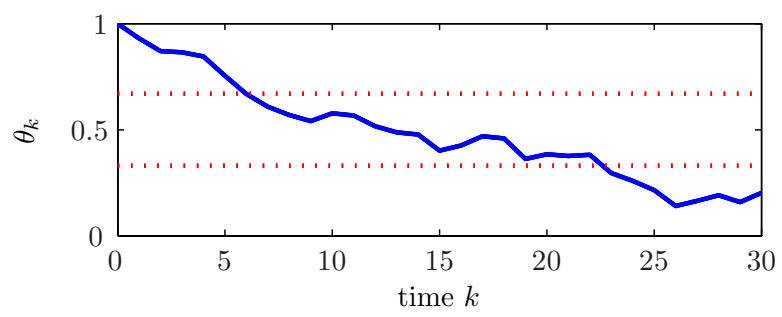

Fig. 2. Scheduling parameter trajectory during the simulations. The dotted lines indicate the boundaries of the parameter simplex regions of brv2.

\section{NUMERICAL EXAMPLE}

This section consists of a numerical example, demonstrating the application of the proposed method and comparing it to robust MPC and the LPV-MPC scheme from Section 3. Knowledge of the future scheduling parameter values can be of crucial importance for control performance. In order to demonstrate this statement, the following example was constructed. Consider the LPV system

$$
x_{k+1}=\left[\begin{array}{cc}
1 & 0 \\
-1+2 \theta_{k} & 0.99
\end{array}\right] x_{k}+\left[\begin{array}{c}
1 \\
-0.2+0.4 \theta_{k}
\end{array}\right] u_{k},
$$

under the constraints

$$
-1 \leq u_{k} \leq 1, \quad\left[\begin{array}{l}
-1 \\
-1
\end{array}\right] \leq x_{k} \leq\left[\begin{array}{l}
1 \\
1
\end{array}\right]
$$

The parameter $\theta_{k}$ is assumed to vary only slowly, with the rate of variation

$$
\Gamma=\{\gamma:-0.1 \leq \gamma \leq 0.1\}
$$

This system can be seen as a disturbed double integrator, where the influence of the first integrator on the second depends on the scheduling parameter. Note that the scheduling parameter has a severe influence on the dynamics of the system.

Three different controllers for this system were compared in terms of complexity and control performance. By considering the scheduling parameter as an unknown, bounded parametric uncertainty, an explicit robust controller was computed, in the following indicated by rob. This robust MPC scheme is presented in detail in Bemporad et al. [2003], where it is derived as the solution to the closed-loop constrained robust optimal (CL-CROC) problem. The resulting control law (6) is parameterindependent, $u_{k}=\mu\left(x_{k}\right)$. Following the procedure in Besselmann et al. [2008b], one explicit LPV-MPC controller with an affine parametrization (8) in the scheduling parameter and the Pólya degree 2 (aff2) was computed. Finally an LPV-MPC controller, which takes the bounded rate of variation (26) into account, $u_{k}=\mu\left(x_{k}, \theta_{k}, \Gamma\right)$ was computed as described in Section 4. The parameter simplex was uniformly divided in three regions, and for each region, a parameter-affine control law was computed using a Pólya degree of 2 - brv2.

The Multi-Parametric Toolbox (MPT) and YALMIP were used to compute the control laws, Kvasnica et al. [2004], Löfberg [2008]. The weight matrices

$$
Q=\left[\begin{array}{ll}
1 & 0 \\
0 & 1
\end{array}\right], \quad R=0.01, \quad P=Q
$$



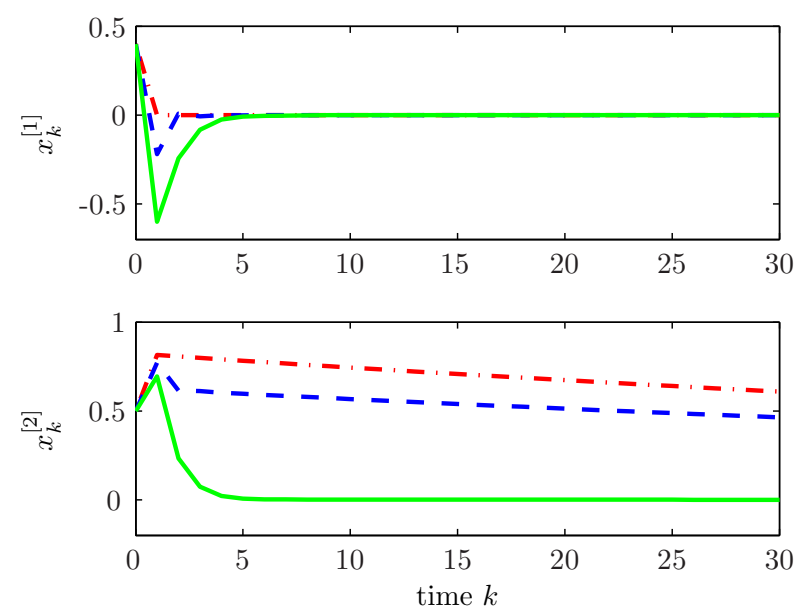

Fig. 3. State trajectories of the LPV system (24) under the control of rob $(-\cdot-)$, aff2 $(--)$ and brv2 (-).

and a prediction horizon of $N=4$ were chosen. The $\infty$ norm was used in the cost function (7).

All control laws were tested in the task to regulate the system from the initial point $x_{0}=[0.4,0.5]$ to the origin, while the scheduling parameter evolves as shown in Figure 2. In Figure 3 the state trajectories of the system controlled by each controller is depicted.

The strategy of the rob, which comprises 5 regions only, is simple. Since even the sign of the influence of the first integrator on the second is uncertain, it discharges the first integrator immediately to prevent a counter-productive influence. Instead rob relies on the stability of the second to eventually converge to the origin. A similar strategy can be observed from aff 2 , which comprises 182 regions. In order to charge the first integrator appropriately, knowledge of the current scheduling parameter is not sufficient. Nevertheless, aff 2 yields a small benefit from that knowledge during the first steps. The crucial point in this example is knowledge of the future scheduling parameter values. When the effect of the first integrator on the second in the succeeding time step is conceivable, the first integrator can be charged accordingly. This allows brv2 (which comprises 520 regions) to outperform the other controllers significantly and to steer the system to the origin within 5 time steps.

\section{REFERENCES}

P. Apkarian, P. Gahinet, and G. Becker. Self-scheduled $H_{\infty}$ control of linear parameter-varying systems: A design example. Automatica, 31(9):1251 - 1261, 1995.

G. Balas, J. Bokor, and Z. Szabó. Invariant subspaces for LPV systems and their applications. IEEE Trans. on Automatic Control, 48(11):2065 - 2069, November 2003.

B. Bank, J. Guddat, D. Klatte, B. Kummer, and K. Tammer. Non-Linear Parametric Optimization. AkademieVerlag, Berlin, 1982.

A. Bemporad and M. Morari. Control of systems integrating logics, dynamics, and constraints. Automatica, 35 (5):407 - 427, 1999.
A. Bemporad, F. Borrelli, and M. Morari. Model predictive control based on linear programming - the explicit solution. IEEE Transactions on Automatic Control, 47 (12):1974 - 1985, December 2002

A. Bemporad, F. Borrelli, and M. Morari. Min-max control of constrained uncertain discrete-time linear systems. IEEE Transactions on Automatic Control, 48(9):1600 - 1606, September 2003.

D. P. Bertsekas. Dynamic programming and optimal control, volume one. Athena Scientific, 1995.

T. Besselmann, J. Löfberg, and M. Morari. Explicit model predictive control for systems with parameter-varying state transition matrix. In IFAC World Congress, Seoul, Korea, 2008a.

T. Besselmann, J. Löfberg, and M. Morari. Explicit model predictive control for linear parameter-varying systems. In Proc. of the Conf. on Decision $\&$ Control, Cancun, Mexico, 2008b.

F. Borrelli. Constrained Optimal Control of Linear and Hybrid Systems, volume 290 of Lecture Notes in Control and Information Sciences. Springer-Verlag, 2003.

L. Chisci, P. Falugi, and G. Zappa. Gain-scheduling MPC for nonlinear systems. Int. Journal of Robust and Nonlinear Control, 13:295 - 308, 2003.

B.N. Delaunay. Sur la sphère vide. Bulletin of Academy of Sciences of the USSR, 7(6):793-800, 1934.

S. Hovland and J.T. Gravdahl. Complexity reduction in explicit MPC through model reduction. In Proceedings of the IFAC World Congress, Seoul, Korea, July 2008.

M. Kvasnica, P. Grieder, M. Baotic, and M. Morari. Multi-Parametric Toolbox (MPT). In Hybrid Systems: Computation and Control, pages 448-462, March 2004.

J. Löfberg. Modeling and solving uncertain optimization problems in YALMIP. IFAC World Congress, 2008.

Y. Lu and Y. Arkun. Quasi-min-max MPC algorithms for LPV systems. Automatica, 36:527 - 540, 2000.

E.N. Pistikopoulos, V. Dua, N.A Bozinis, A. Bemporad, and M. Morari. On-line Optimization via Off-line Parametric Optimization Tools. In International Symposium on Process Systems Engineering, pages 183-188, Keystone, USA, July 2000.

G. Pólya. Über positive Darstellung von Polynomen. Vierteljahrschrift $d$. naturforschenden Gesellschaft in Zürich, 73:141 - 145, 1928. reprinted in: Collected Papers, Volume 2, 309 - 313, Cambridge: MIT Press, 1974.

V. Powers and B. Reznick. A new bound for Pólya's theorem with applications to polynomial positive on polyhedra. Journal of Pure and Applied Algebra, 164: $221-229,2001$.

J.S. Shamma and M. Athans. Analysis of gain scheduled control for nonlinear plants. IEEE Trans. on Automatic Control, AC-35:898 - 907, 1990.

J.S. Shamma and M. Athans. Guaranteed properties of gain scheduled control for linear parameter-varying plants. Automatica, 27:559 - 564, 1991.

L.M. Silverman and H.E. Meadows. Controllability and observability in time-variable linear systems. SIAM Journal on Control, 5(1):64 - 73, 1967. doi: $10.1137 / 0305005$. 